\title{
Spatio-Temporal Chaos in Simple Coupled Chaotic Circuits
}

\author{
Yoshifumi Nishio, Member, IEEE, and Akio Ushida, Membèr, IEEE
}

\begin{abstract}
In this paper, simple autonomous chaotic circuits coupled by resistors are investigated. By carrying out computer calculations and circuit experiments, irregular self-switching phenomenon of three spatial patterns characterized by the phase states of quasi-synchronization of chaos can be observed from only four simple chaotic circuits. This is the same phenomenon as chaotic wandering of spatial patterns observed very often from systems with a large number of degrees of freedom. Namely, one of spatial-temporal chaos observed from systems of large size can be also generated in the proposed system consisting of only four chaotic circuits. A six subcircuits case and a coupled chaotic circuits networks are also studied, and such systems are confirmed to produce more complicated spatio-temporal phenomena.
\end{abstract}

\section{INTRODUCIION}

$\mathbf{M}$ ANY NONLINEAR dynamical systems in the various fields have been clarified to exhibit chaotic oscillations, and recently, applications of chaos to engineering systems attract many researchers' attentions, for example, chaos noise generator, control of chaos, synchronization of chaos, and so on. Among the studies on such applications, synchronization of chaotic systems or signals is extremely interesting [1]-[3], because the chaotic solution is unstable, and small error of initial values must be expanded as time goes. As far as we know, such phenomena have been first reported to be generated in simple real circuits by a group of Saito [1]. Since Pecora et al. have investigated such phenomena theoretically [2], many papers have been published until now. We have proposed coupled chaotic circuits generating various types of quasi-synchronizations [4]-[7].

On the other hand, a network of chaotic one-dimensional maps has been investigated earnestly by Kaneko [8]-[11]. $\mathrm{He}$ has discovered various kinds of phenomena called spatiotemporal chaos such as diffusion and Brownian motion of defect, clustering, spatio-temporal intermittency, and so on. The study of such systems are very important not only as models for nonlinear systems with many degrees of freedom but also for the clarification of biological information processing [12] and for engineering applications. Actually, Aihara $e t$ al. have proposed chaotic ncural networks whose cell is a onedimensional chaotic map, and they have confirmed that such systems produce dynamical chaotic search for memorized patterns [13], [14]. Recently, Chua and his colleagues published

Manuscript received January 19, 1995; revised June 8, 1995. This paper was recommended by Guest Editor L. O. Chua.

The authors are with the Department of Electrical and Electronic Engineering, Tokushima University, Tokushima, 770 Japan.

IEEE Log Number 9414464. their papers on spatio-temporal chaos observed in a chain of coupled Chua's circuits, which are real physical continuous systems [15]-[17]. For the purpose of the clarification of various phenomena in natural fields, it is very important to investigate spatial-temporal phenomena observed from such real physical circuit models.

In this paper, we investigate four simple autonomous chaotic circuits coupled by one resistor. This is a four subcircuits case of the circuit proposed in [7]. In [7], we briefly mentioned the generation of some phenomena discussed in this paper. By carrying out computer calculations and circuit experiments, irregular self-switching phenomenon of three spatial patterns characterized by the phase states of quasi-synchronization of chaos can be observed. The irregularity of the change of spatial patterns is investigated by using the Poincaré map. This is the same phenomenon as chaotic wandering of spatial patterns observed very often from systems with a large number of degrees of freedom. Namely, one of spatial-temporal chaos observed from systems of large size can be also generated in the proposed system consisting of only four chaotic circuits. Usually, the term "spatio-temporal chaos" is used for irregular dynamical phenomena observed from a large number of coupled chaotic systems that is spatially extensive. However, there are many spatial-temporal phenomena observed from both the large size and small size of coupled systems, for example, chaotic wandering of spatial patterns discussed in this paper, clustering of spatially arranged chaotic cells, and so on. In such cases, we consider that the basic mechanism of the generation of some spatial-temporal phenomena is almost the same in spite of the system size. Namely, the number of chaotic cells is not essential, and it seems to be better to analyze simple systems of small size first and to extend the study to systems of large size with reference to the results obtained from small size. If observed phenomena are almost the same, we had better not make distinctions between systems of large size and small size. Because the most important subject is clarification of observed phenomena. Hence, we use the term "spatio-temporal chaos" in this paper as irregular dynamical changes of spatial patterns observed from coupled chaotic systems arranged spatially. Please note we pay attention to the phenomena without restriction on the size of systems. We consider that the irregular self-switching phenomenon of three spatial patterns observed from the four chaotic circuits is one of the simplest spatio-temporal chaos (in the sense of abovementioned definition) observed from continuous dynamical systems. 

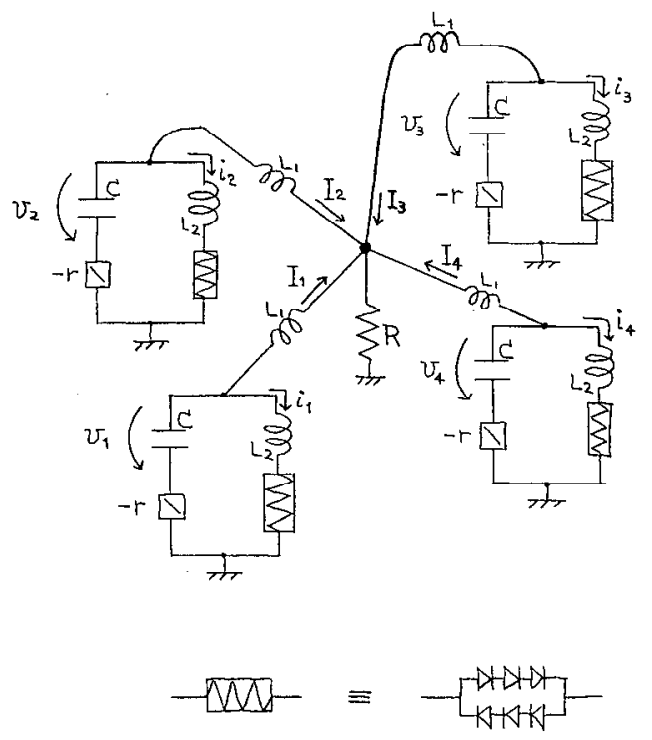

Fig. 1. Basic circuit model.

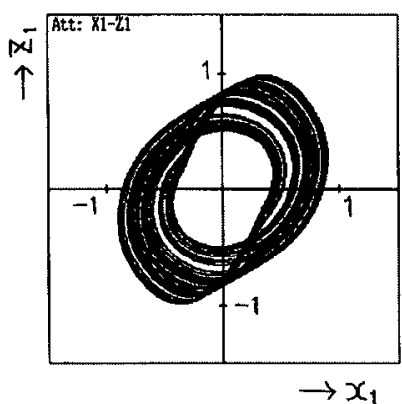

(a)

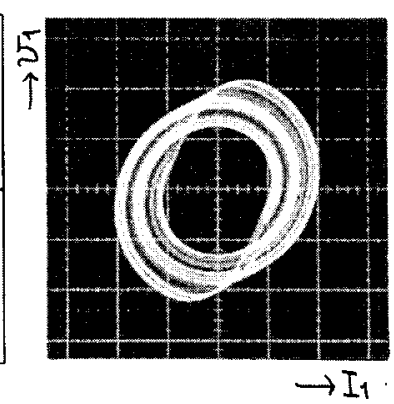

(b)
Fig. 2. Typical example of chaotic attractors observed from the chaotic subcircuit. (a) Computer calculation for $\alpha=24.0$ and $\beta=0.295$. (b) Circuit experiment for $L_{1}=204.15 \mathrm{mH}, L_{2}=9.933 \mathrm{mH}, C=0.03425 \mu \mathrm{F}$, and $r=630 \Omega$. Horizontal: $0.4 \mathrm{~mA} /$ div. Vertical: $1 \mathrm{~V} / \mathrm{div}$.

We also study on the six subcircuits case as an extended example and confirm the generation of more complicated irregular self-switching phenomenon of spatial patterns. Moreover, in order to show the possibility of the extension to systems of large size, we carry out computer simulation for a $3 \times 3$ network based on the coupled oscillators network [18] and confirm the generation of the chaotic wandering of many spatial patterns. This result also indicates that the self-switching phenomenon observed from only four chaotic circuits is the same as the chaotic wandering observed from systems of large size.

\section{BASIC CIRCUIT MODEL}

Basic circuit model is shown in Fig. 1. Please note that this is a four subcircuits case of the circuit proposed in [7]. In this system, four of the same chaotic circuits are coupled by one resistor $R$. When the coupling resistor $R$ is equal to zero, all $L_{1}$ 's are grounded, and four subcircuits become completely independent. Each chaotic subcircuit is a symmetric version of the circuit model proposed by Inaba $e t a l$. [19]. It consists of three elements, one linear negative resistor, and one nonlinear resistor, which is realized by connecting some diodes, and is one of the simplest chaotic circuits. We have confirmed that this subcircuit exhibits the bifurcation phenomena that are similar to those reported in [20]. By using the same method in [20], we will be able to prove the generation of logistic chaos and two types of windows from the subcircuit. Fig. 2 shows a typical example of chaotic attractors obtained from the uncoupled chaotic subcircuit. In the following circuit experiments, the values of the inductors and the capacitor in each chaotic subcircuit are fixed, and those values are measured as $L_{1}=204.15 \mathrm{mH} \pm 0.073 \%, L_{2}=9.933 \mathrm{mH}$ $\pm 0.030 \%$, and $C=0.03425 \mu \mathrm{F} \pm 0.29 \%$.

At first, we approximate the $i-v$ characteristics of the nonlinear resistor consisting of diodes by the following function.

$$
v_{d}\left(i_{k}\right)=\sqrt[9]{r_{d} i_{k}}
$$

By changing the variables and parameters,

$$
\begin{aligned}
& t=\sqrt{L_{1} C \tau}, \quad I_{k}=a \sqrt{\frac{C}{L_{1}}} x_{k}, \quad i_{k}=a \sqrt{\frac{C}{L_{1}}} y_{k}, \\
& v_{k}=a z_{k}, \\
& \alpha=\frac{L_{1}}{L_{2}}, \quad \beta=r \sqrt{\frac{C}{L_{1}}}, \quad \gamma=R \sqrt{\frac{C}{L_{1}}}, \quad \cdots . "=\frac{d}{d \tau}, \\
& \quad\left(\text { where } a=\sqrt[8]{r_{d} \sqrt{\frac{C}{L_{1}}}}\right)
\end{aligned}
$$

the circuit equation is normalized as

$$
\begin{aligned}
& \dot{x}_{k}=\beta\left(x_{k}+y_{k}\right)-z_{k}-\gamma \sum_{j=1}^{N} x_{j} \\
& \dot{y}_{k}=\alpha\left\{\beta\left(x_{k}+y_{k}\right)-z_{k}-f\left(y_{k}\right)\right\} \\
& \dot{z}_{k}=x_{k}+y_{k} \quad(k=1,2,3,4)
\end{aligned}
$$

where

$$
f\left(y_{k}\right)=\sqrt[9]{y_{k}} .
$$

Please note that when the coupling parameter $\gamma$, which is proportioned to $R$, is equal to zero, the coupling term in (3) vanishes.

For computer calculations, in order to consider the difference of real circuit elements, (3) is rewritten using an additional parameter $\Delta \omega$ as follows:

$$
\begin{aligned}
\dot{x}_{k} & =\beta\left(x_{k}+y_{k}\right)-z_{k}-\gamma \sum_{j=1}^{N} x_{j} \\
\dot{y}_{k} & =\alpha\left\{\beta\left(x_{k}+y_{k}\right)-z_{k}-f\left(y_{k}\right)\right\} \\
\dot{z}_{k} & =\{1+(k-1) \Delta \omega\}\left(x_{k}+y_{k}\right) \quad(k=1,2,3,4) .
\end{aligned}
$$

In the following computer calculations, we fix the parameter $\alpha$ as 24.0 and (5) is calculated by using the Runge-Kutta method with step size $\Delta t=0.01$. 

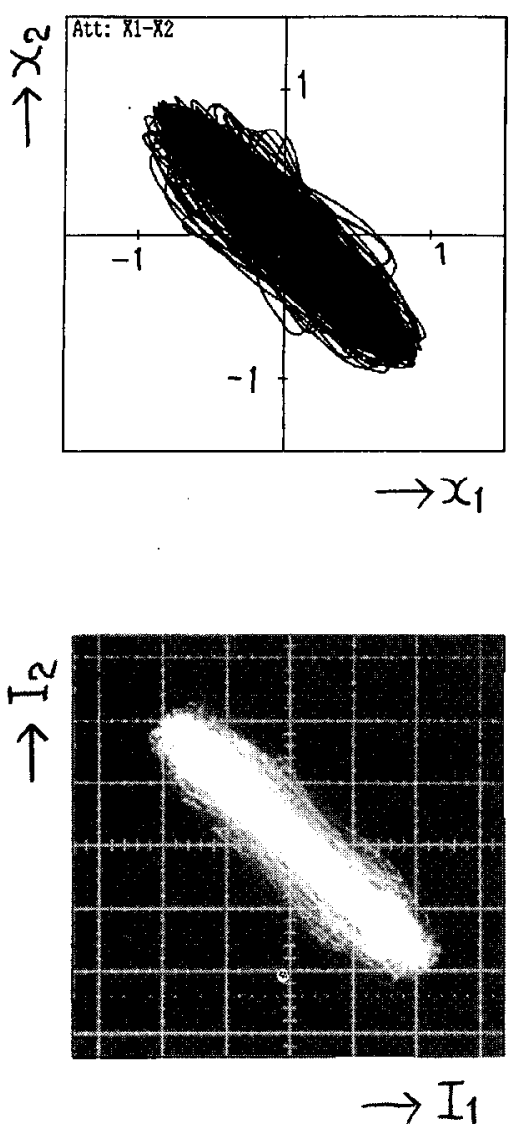

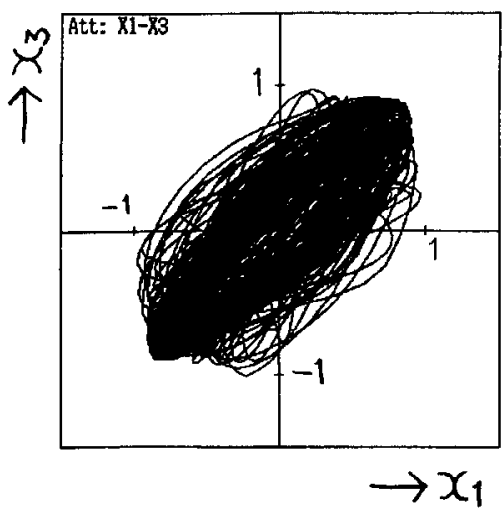

(a)
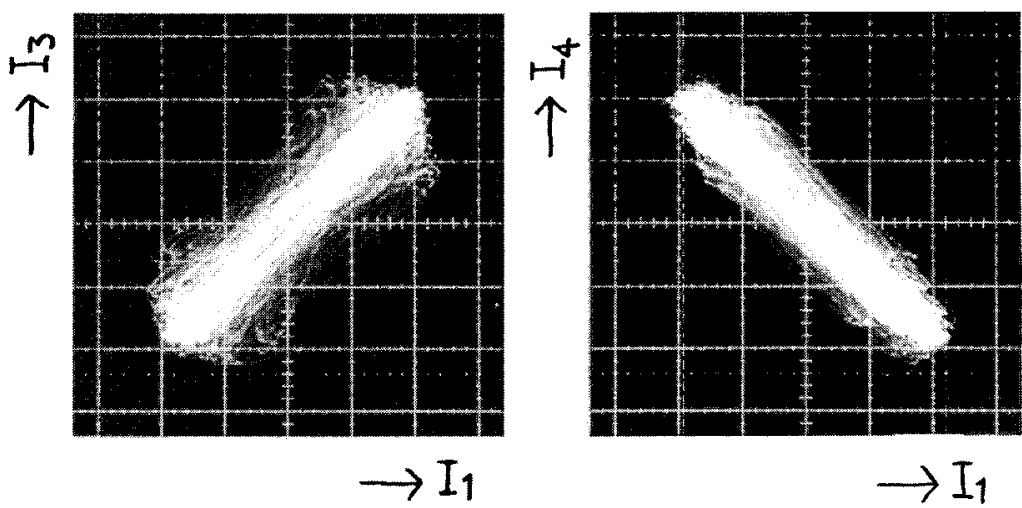

(b)

Fig. 3. In- and opposite-phases quasi-synchronization (4 subcircuits). (a) Computer calculation for $\beta=0.29, \gamma=0.40$, and $\Delta \omega=0.0$. (b) Circuit experiment for $r=570 \Omega$ and $R=2.4 \mathrm{k} \Omega$. Horizontal and Vertical: $0.4 \mathrm{~mA} / \mathrm{div}$.

\section{QUASI-SYNCHRONIZATION OF CHAOS}

Before we treat spatio-temporal chaos, we introduce two types of quasi-synchronizations of chaos in this section. We use the term quasi-synchronization only for qualitative explanation of the observed phenomena. The term means that phase shift between two chaotic signals is not completely constant but almost within some relatively small range. The generation of these quasi-synchronization phenomena have been already reported in [7]. Because these phenomena are deeply related with spatio-temporal chaos in the next section, we show some examples briefly.

The first type is the in- and opposite-phases quasisynchronization. Fig. 3 shows an example of the in- and opposite-phases quasi-synchronizations. In this case, each subcircuit exhibits chaos as Fig. 2. But, two of the four subcircuits are almost synchronized at the in-phase, and the rest is almost synchronized to the two subcircuits with $\pi$ phase difference. Namely, phase difference with respect to the subcircuit 1 is described as $\{0, \pi, 0, \pi\}$ for the example in Fig. 3. Though we omit other phase states in Fig. 3, there coexists more two different phase states, namely $\{0,0, \pi, \pi\}$ and $\{0, \pi, \pi, 0\}$.

The second type is the two pairs of opposite-phases quasisynchronization. Fig. 4 shows an example of the two pairs of opposite-phases quasi-synchronizations. In this case, subcircuits 1 and 2 are almost synchronized at the opposite-phase. Also, subcircuits 3 and 4 are almost synchronized at the opposite-phase. However, a pair of subcircuits 1-2 and the other pair of 3-4 are independent. We had considered that there coexists more two different phase states, namely $\{1-3,2-4\}$ and $\{1-4,2-3\}$. However the combination of the decoupling into two pairs is decided by the slight difference of real circuit clements, and other combination states cannot be observed. Namely, this quasi-synchronization is based on the asymmetry of the coupling and cannot be generated in the case of completely symmetric coupling as $\Delta \omega=0.0$. We consider that this phenomenon is deeply related with the clustering [11]. However, in this paper, this phenomenon is not treated as spatio-temporal chaos because the spatial pattern is always the same.

\section{Spatio-Temporal Chaos}

For a large region in parameter space, we observed complex chaotic motion. Namely, we observed that three phase states of the in- and opposite-phases quasi-synchronizations are switched automatically and randomly as shown in Fig. 5. The order of the appearance of three phase states is truly unpredictable. Further switching period is also chaotic, namely 

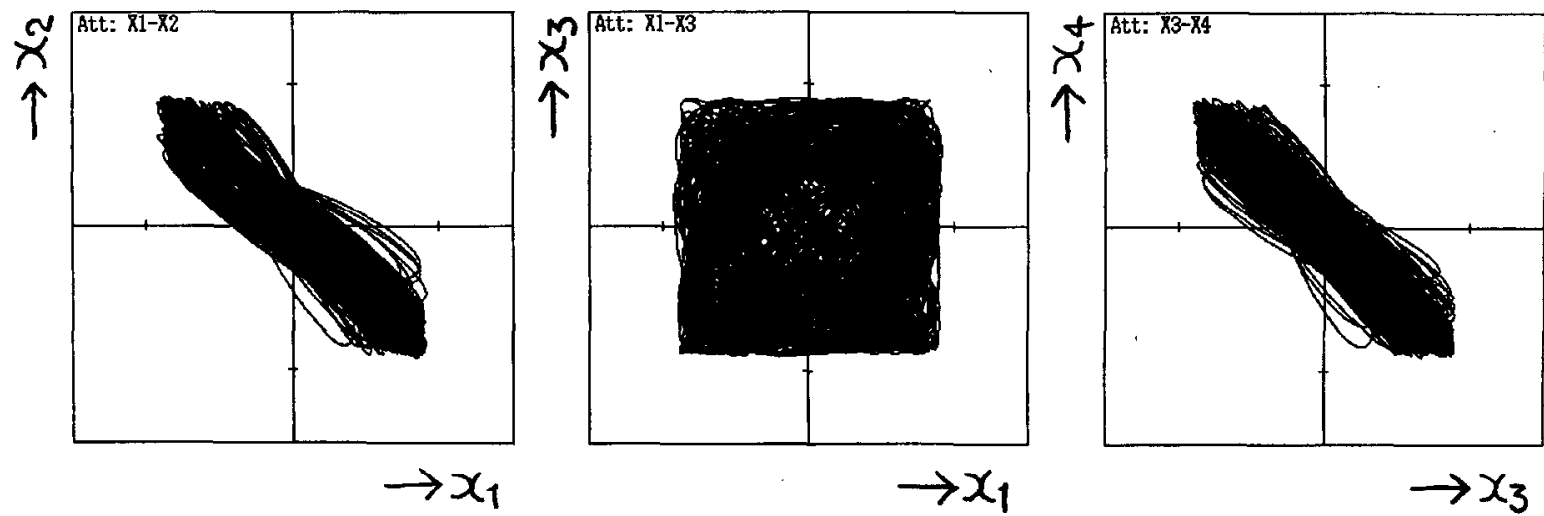

(a)
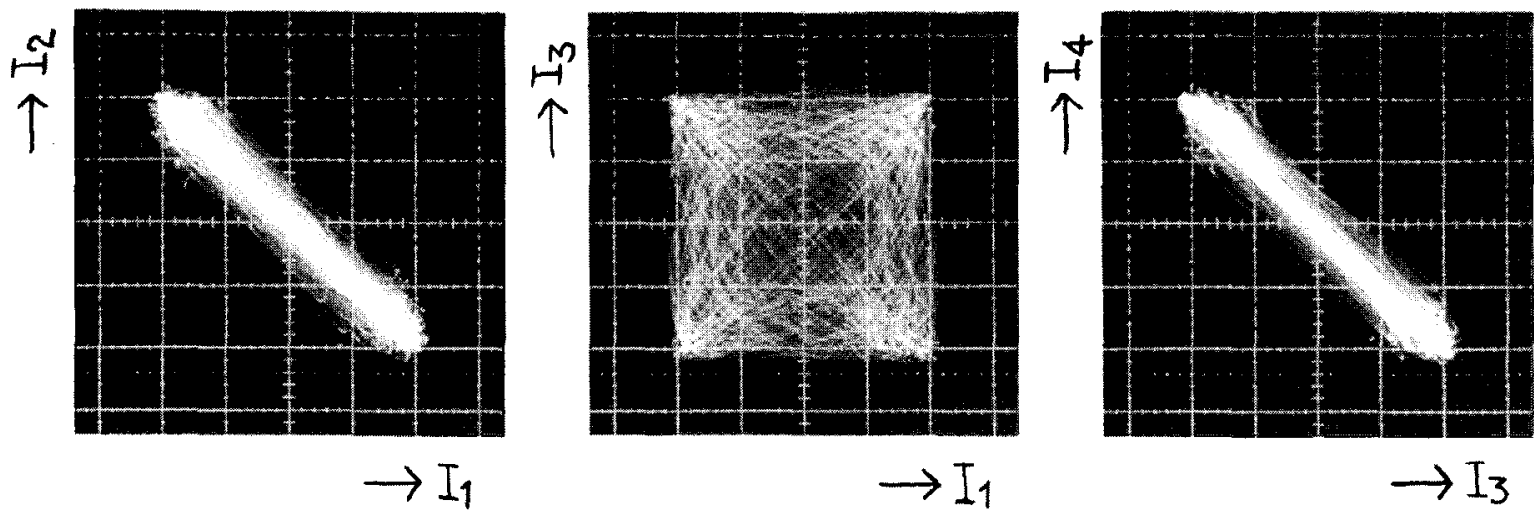

(h)

Fig. 4. Two pairs of opposite-phases quasi-synchronization (4 subcircuits). (a) Computer calculation for $\beta=0.295, \gamma=0.34$, and $\Delta \omega=0.01$. (b) Circuit experiment for $r=580 \Omega$ and $R=850 \Omega$. Horizontal and Vertical: $0.4 \mathrm{~mA} /$ div.

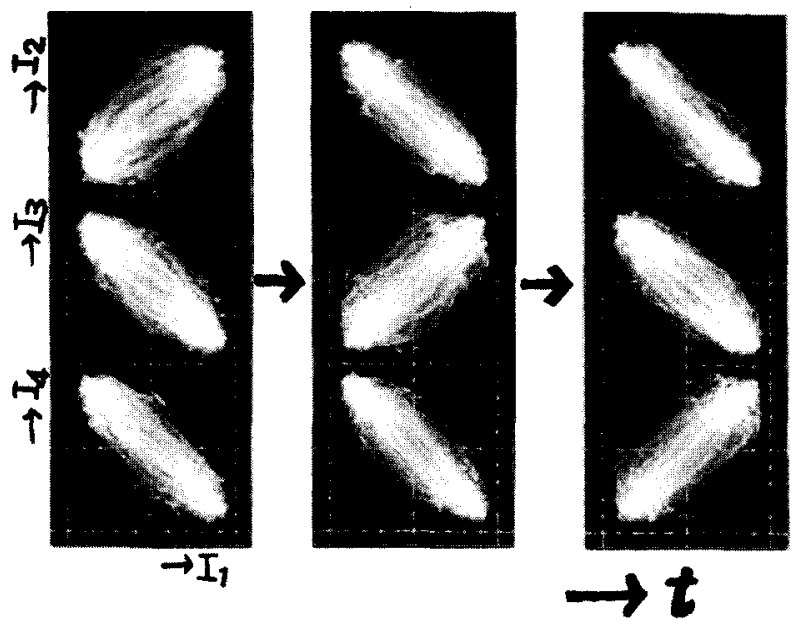

Fig. 5. Spatio-temporal chaos: Self-switching of in- and opposite-phases quasi-synchronization (4 subcircuits). $r=630 \Omega$ and $R=1.3 \mathrm{k} \Omega$. Horizontal and Vertical: $1.0 \mathrm{~mA} / \mathrm{div}$.

a state may be switched to the next state instantly, and a state may be switched after about a few seconds. We also observed similar self-switching phenomenon of three phase states of the two pairs of opposite-phases quasi-synchronizations as shown in Fig. 6. This is the same phenomenon as chaotic wandering

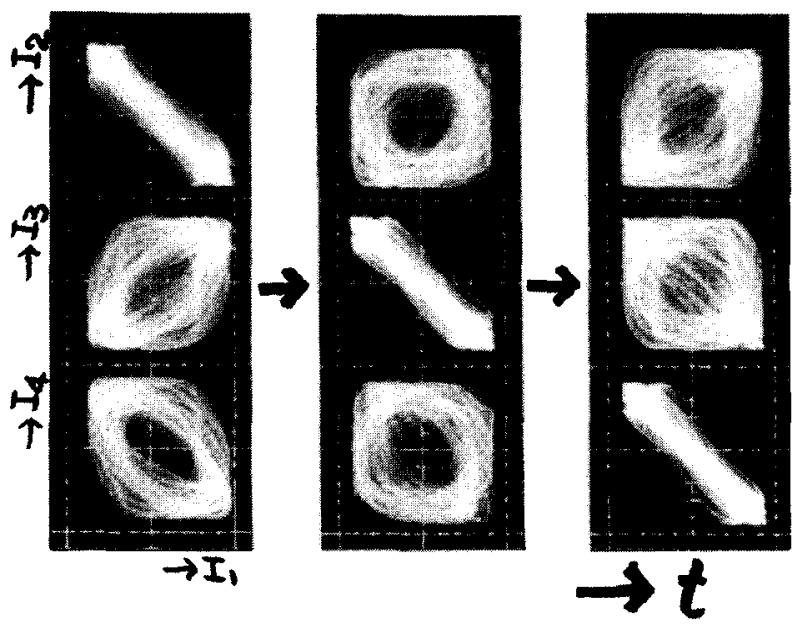

Fig. 6. Spatio-temporal chaos: Self-switching of two pairs of opposite-phases quasi-synchronization (4 subcircuits). $r=630 \Omega$ and $R=96 \Omega$. Horizontal and Vertical: $1.0 \mathrm{~mA} / \mathrm{div}$.

of spatial patterns observed very often from systems with a large number of degrees of freedom. The unpredictability of chaotic wandering is caused by local chaotic motions. Because we cannot predict low-dimensional chaotic motion, the change caused by the chaotic motion of subcircuits is also 


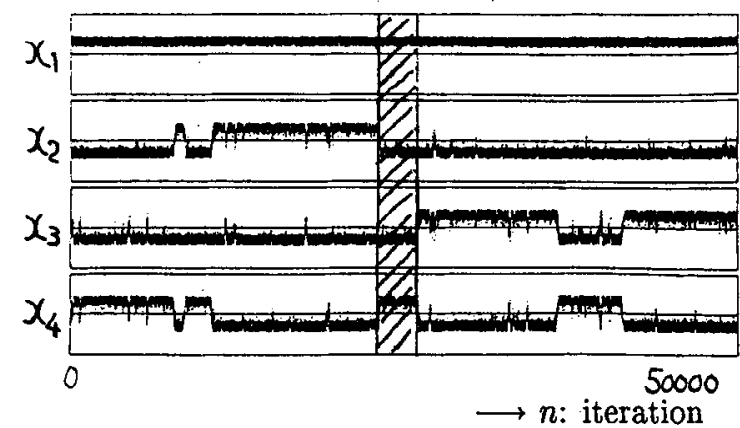

Fig. 7. Time series of Poincaré map: Self-switching of in- and opposite-phases quasi-synchronization (4 subcircuits). $\beta=0.295, \gamma=0.10$, and $\Delta \omega=0.0$.

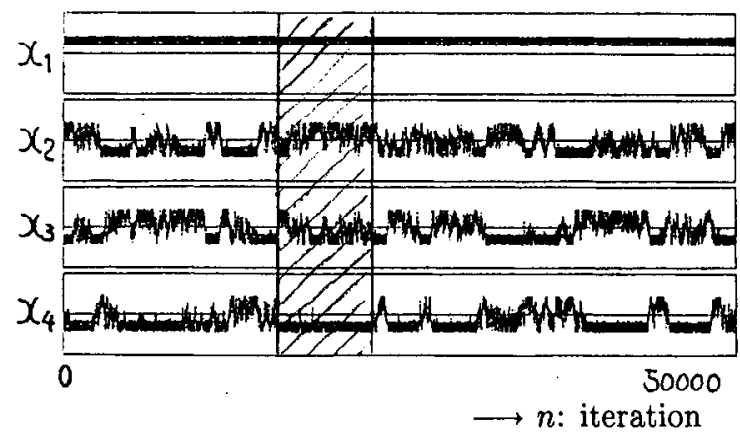

Fig. 8. Time series of Poincaré map: Self-switching of two pairs of opposite-phases quasi-synchronization (4 subcircuits). $\beta=0.295, \gamma=0.01$, and $\Delta \omega=0.0$.

unpredictable. We treat this phenomenon as one of spatiotemporal chaos. Because three spatial patterns characterized by phase states of quasi-synchronization of chaos changes irregularly as time goes, and it is caused by the local chaotic motion of subcircuits arranged spatially. The physical mechanism of the generation of self-switching will be explained as follows. In the case that unstability of the chaotic solution from each subcircuit is very strong, the current flowing into the coupling resistor $R$ will often take relatively large value. It influences the motion of the solution in other subcircuits via coupling resistor $R$ and at the moment the quasi-synchronization state will be disturbed. The self-switching is considered to be generated when the disturbance happens to act to switch the phase states of two of the four subcircuits.

In order to investigate this phenomenon, we define the Poincaré section as $z_{1}=0$ where $d z_{1} / d t>0$ and plot the values of $x_{i}(i=1,2,3,4)$ on $x_{i}-n$ ( $n$ denotes the number of iterations of the Poincare map) plane when the solution hits the Poincare section. Fig. 7 shows the time series of attractors corresponding to the self-switching of the in- and opposite-phases quasi-synchronizations obtained from computer simulations. For example, the phase state of $\{0, \pi, \pi, 0\}$ appears in the shaded area. In the figure, we can confirm that three phase states appear chaotically.

Fig. 8 shows the time series of attractors corresponding to the self-switching of the two pairs of opposite-phases quasisynchronizations obtained from computer simulations. For example, the phase state of $\{1-4,2-3\}$ appears in the shaded

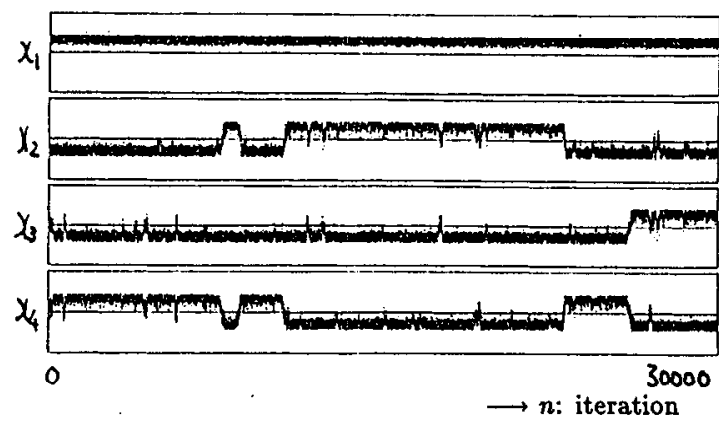

(a)

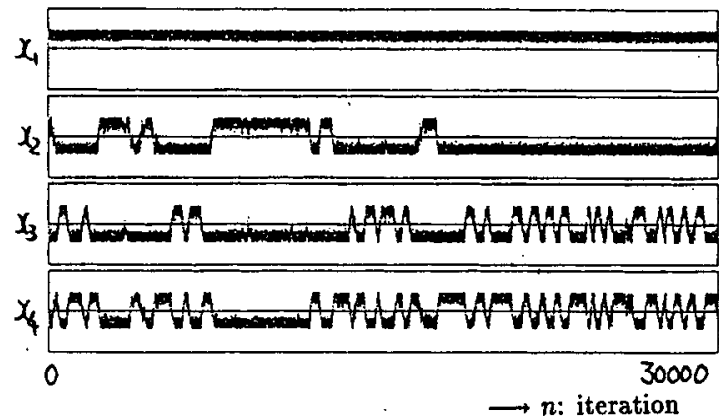

(b)

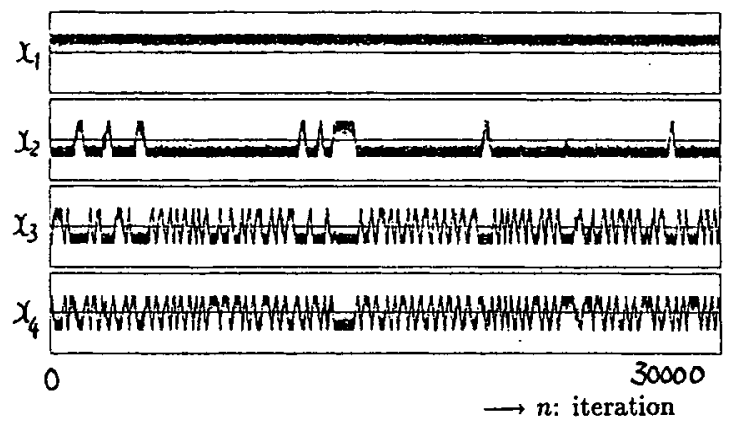

(c)

Fig. 9. Effect of the value of $\Delta \omega$ (4 subcircuits). $\beta=0.295$ and $\gamma=0.10$. (a) $\Delta \omega=0.000$ (same as Fig. 7). (b) $\Delta \omega=0.003$. (c) $\Delta \omega=0.005$.

area. In the figure, we can confirm that three phase states appear chaotically.

Fig. 9 shows the effect of $\Delta \omega$, namely asymmetry of the circuit. Though we have not investigated detailed statistical characteristics, we can see that both of the appearing frequencies for some pattern and switching speed are deeply related with the value of $\Delta \omega$.

\section{SIX SubCIRCUITS CASE}

In this section, we consider the six subcircuits case as an extended example. The coupling structure is the same as Fig. 1 except that the six $L_{1}$ 's from the six subcircuits are connected to the coupling resistor $R$ located in the center. We can expect the generation of more complicated irregular self-switching of phase states.

Fig. 10 shows an example of the in- and opposite-phases quasi-synchronization. In the six subcircuits case, there 

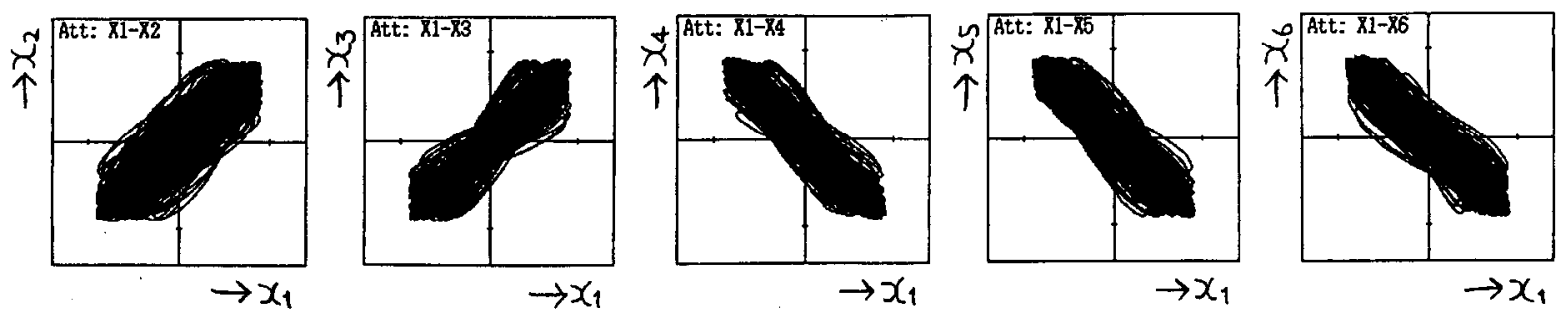

Fig. 10. In- and opposite-phases quasi-synchronization (6 subcircuits). $\beta=0.29, \gamma=0.10$, and $\Delta \omega=0.0$.

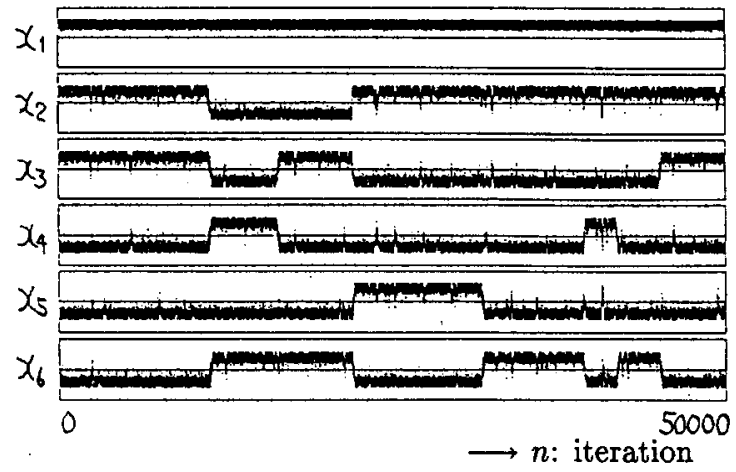

Fig. 11. Time series of Poincaré map: Self-switching of in- and opposite-phases quasi-synchronization ( 6 subcircuits). $\beta=0.294, \gamma=0.07$, and $\Delta \omega=0.0$.

coexists ${ }_{5} C_{2}=10$ phase states. The phase state in the figure is described as $\{0,0,0, \pi, \pi, \pi\}$.

Moreover, the number of the phase states for the three pairs of opposite-phases quasi-synchronization will be ${ }_{6} C_{2}{ }_{4} C_{2} /{ }_{3} P_{3}=15$. Though this type of quasi-synchronization seems to be unstable, we can observe these phase states as switching patterns in spatio-temporal chaos.

Fig. 11 shows the self-switching of the in- and oppositephases quasi-synchronizations obtained from computer simulations. We can see six different phase states in the figure.

Fig. 12 shows the self-switching of the three pairs of opposite-phases quasi-synchronizations obtained from computer simulations. For example, the phase state of $\{1-3,2-4,5-6\}$ appears in the shaded area.

We consider that $2 N$ subcircuits can exhibit spatio-temporal chaos as shown in this section. Because the number of phase states increases for large $N$, more complicated irregular selfswitching will be observed.

\section{COUPLED ChaOtIC CiRCUITS Network}

In this section, we develop the simple coupled chaotic circuit in Fig. 1 to coupled chaotic circuits network. We have already proposed coupled oscillators network consisting of van der Pol oscillators coupled by resistors as in Fig. 13 [18] and have confirmed the generation of various phase states. Based on the study, we propose the chaotic circuits network with resistor coupling.

At first, let us consider the number of frozen spatial patterns corresponding to the in- and opposite-phases quasisynchronization or the two pairs of opposite-phases quasi-

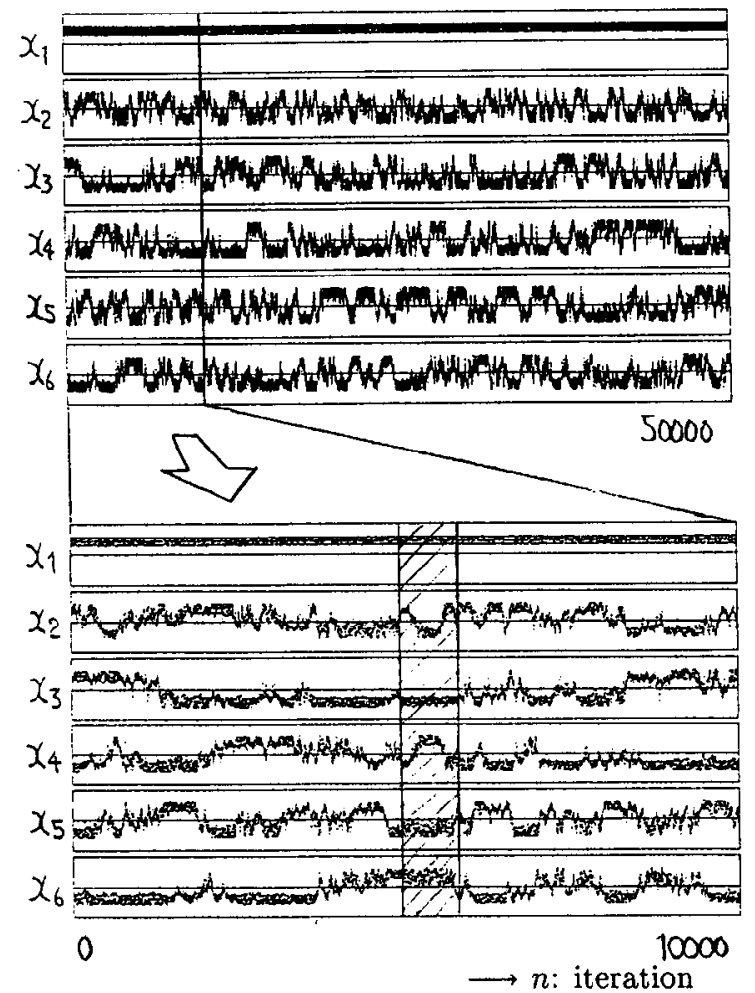

Fig. 12. Time series of Poincaré map: Self-switching of three pairs of opposite-phases quasi-synchronization (6 subcircuits). $\beta=0.295, \gamma=0.01$, and $\Delta \omega=0.0$.

synchronization. If the in- and opposite-phases quasisynchronization is generated around four coupling resistors, the number of the spatial pattern is seven as follows:

$$
\begin{aligned}
& \left(\begin{array}{lll}
0 & 0 & 0 \\
\pi & \pi & \pi \\
0 & 0 & 0
\end{array}\right),\left(\begin{array}{lll}
0 & \pi & 0 \\
0 & \pi & 0 \\
0 & \pi & 0
\end{array}\right),\left(\begin{array}{ccc}
0 & \pi & 0 \\
0 & \pi & 0 \\
\pi & 0 & \pi
\end{array}\right), \\
& \left(\begin{array}{lll}
0 & 0 & \pi \\
\pi & \pi & 0 \\
0 & 0 & \pi
\end{array}\right),\left(\begin{array}{lll}
0 & \pi & \pi \\
\pi & 0 & 0 \\
0 & \pi & \pi
\end{array}\right),\left(\begin{array}{lll}
0 & \pi & 0 \\
\pi & 0 & \pi \\
\pi & 0 & \pi
\end{array}\right), \\
& \left(\begin{array}{lll}
0 & \pi & 0 \\
\pi & 0 & \pi \\
0 & \pi & 0
\end{array}\right)
\end{aligned}
$$

where 0 or $\pi$ means the phase difference with respect to subcircuit 1 . The number of this type of spatial pattern for the size of $N_{1} \times N_{2}$ can be calculated as $2^{N_{1}-1}+2^{N_{2}-1}-1$ using the scaling technique. 

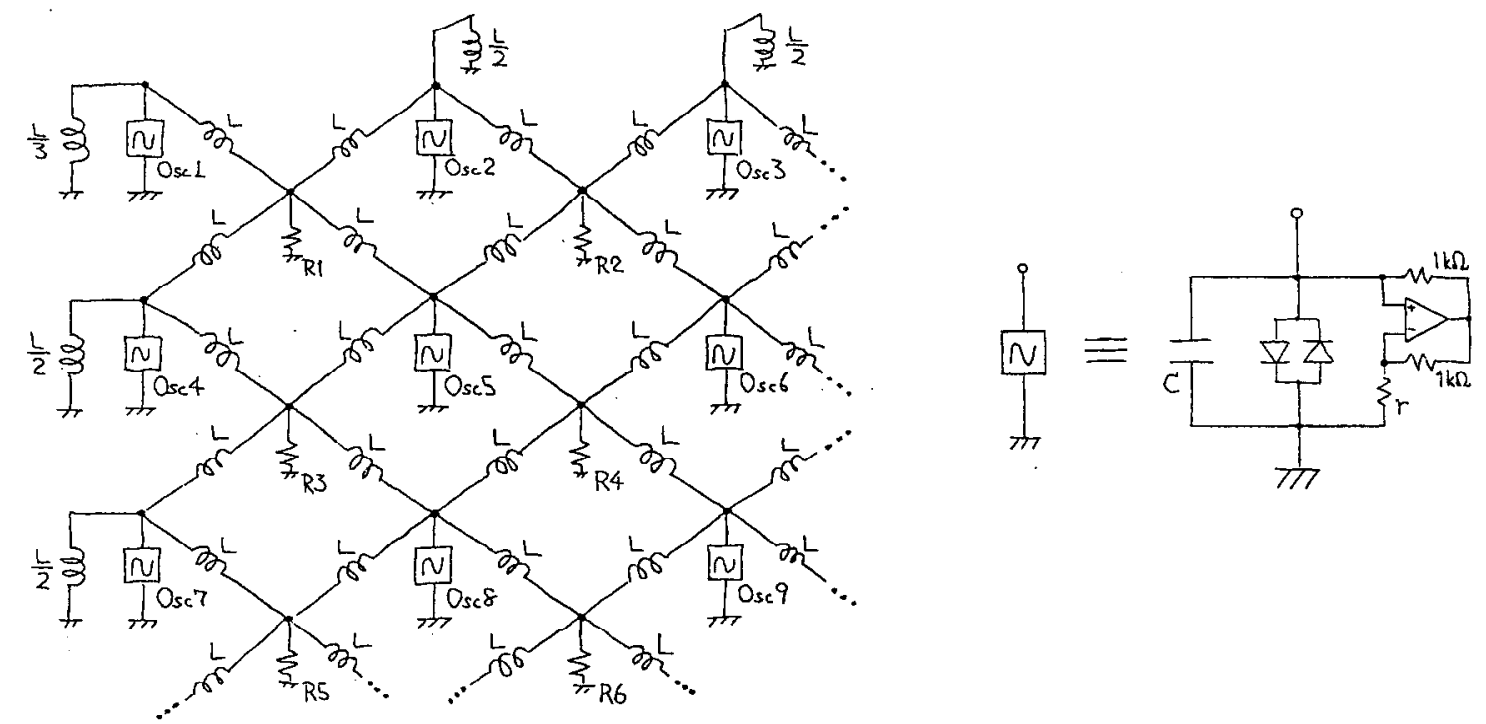

Fig. 13. Coupled oscillators network.
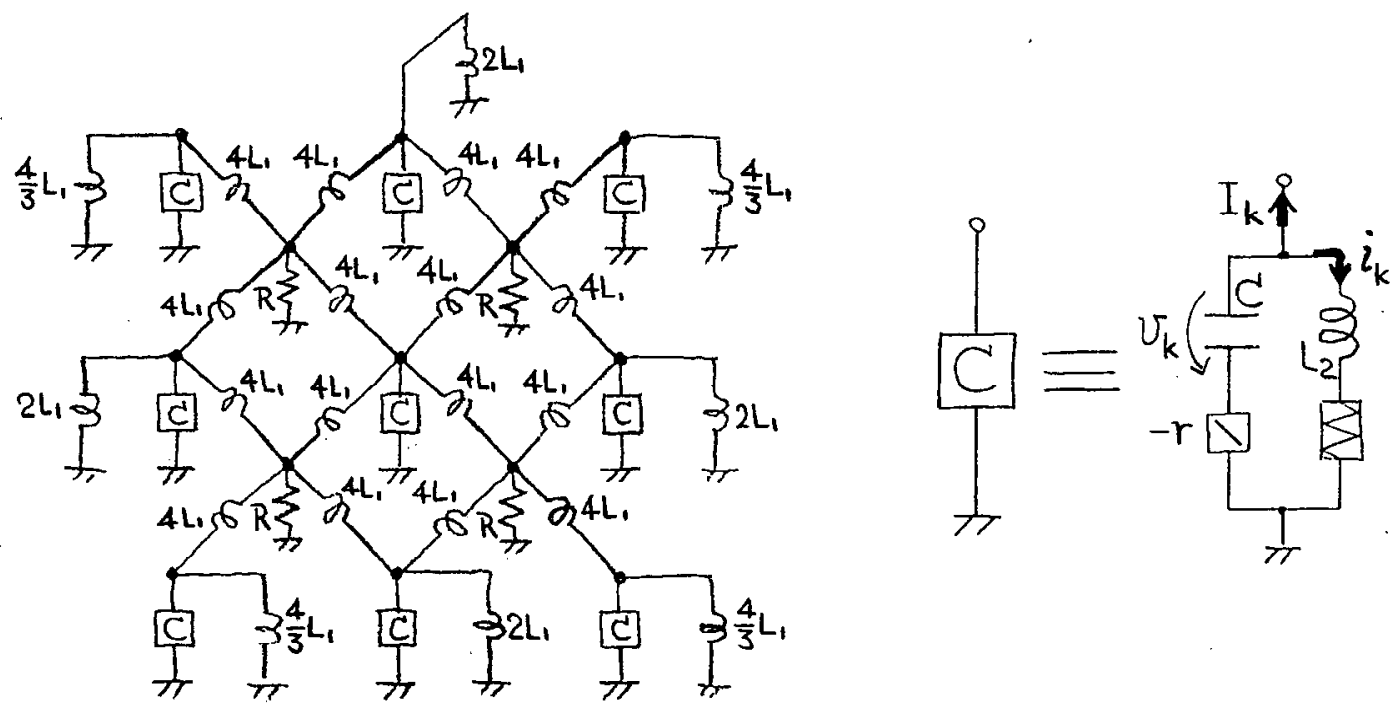

Fig. 14. Coupled chaotic circuits network.

While if the two pairs of opposite-phases quasisynchronization are generated around four coupling resistors, the number of the spatial pattern is also seven as follows:

$$
\begin{aligned}
& \left(\begin{array}{lll}
A & \bar{A} & A \\
B & \bar{B} & B \\
C & \bar{C} & C
\end{array}\right),\left(\begin{array}{ccc}
A & B & C \\
\bar{A} & \bar{B} & \bar{C} \\
A & B & C
\end{array}\right),\left(\begin{array}{ccc}
\bar{A} & \bar{B} & \frac{A}{A} \\
B & A & B
\end{array}\right), \\
& \left(\begin{array}{llll}
A & \bar{A} & B \\
B & \bar{B} & A \\
A & \bar{A} & B
\end{array}\right),\left(\begin{array}{lll}
\bar{A} & \bar{B} & \bar{B} \\
\bar{B} & \bar{A} & A \\
A & B & \bar{B}
\end{array}\right),\left(\begin{array}{ccc}
\bar{A} & \bar{B} & \bar{A} \\
\bar{B} & \bar{B} \\
B & A & B
\end{array}\right), \\
& \left(\begin{array}{lll}
A & B & A \\
\bar{B} & \bar{A} & \bar{B} \\
A & B & A
\end{array}\right)
\end{aligned}
$$

where different characters represent independent phase, and $\bar{A}$ means the opposite phase of $A$.
We investigate the network with the size of $3 \times 3$ shown in Fig. 14. Though this size is the smallest, and wave phenomena cannot be seen, this small network can produce spatio-temporal chaos.

Fig. 15 shows the self-switching of the spatial patterns represented in (6) obtained from computer simulations where the position of subcircuits corresponding to $x_{1} \sim x_{9}$ is as follows:

$$
\left(\begin{array}{lll}
x_{1} & x_{2} & x_{3} \\
x_{4} & x_{5} & x_{6} \\
x_{7} & x_{8} & x_{9}
\end{array}\right)
$$

For example, phase states of

$$
\left(\begin{array}{lll}
0 & \pi & 0 \\
0 & \pi & 0 \\
0 & \pi & 0
\end{array}\right) \text { and }\left(\begin{array}{ccc}
0 & \pi & 0 \\
\pi & 0 & \pi \\
\pi & 0 & \pi
\end{array}\right)
$$

appear in the shaded areas I and II, respectively. As we can see from Fig. 15, the change of spatial patterns observed from the 


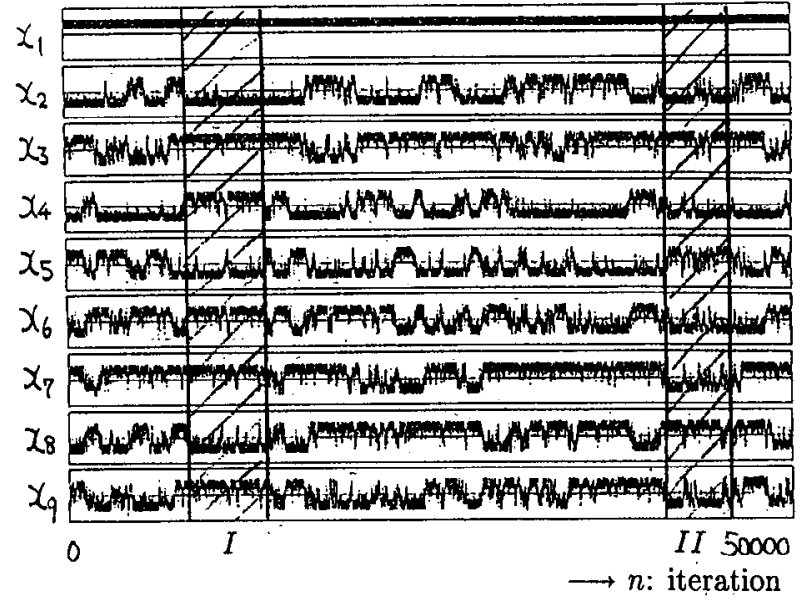

Fig. 15. Time series of Poincaré map: Self-switching of seven spatial patterns in (6) $(3 \times 3$ network). $\beta=0.30, \gamma=0.10$, and $\Delta \omega=0.0$.

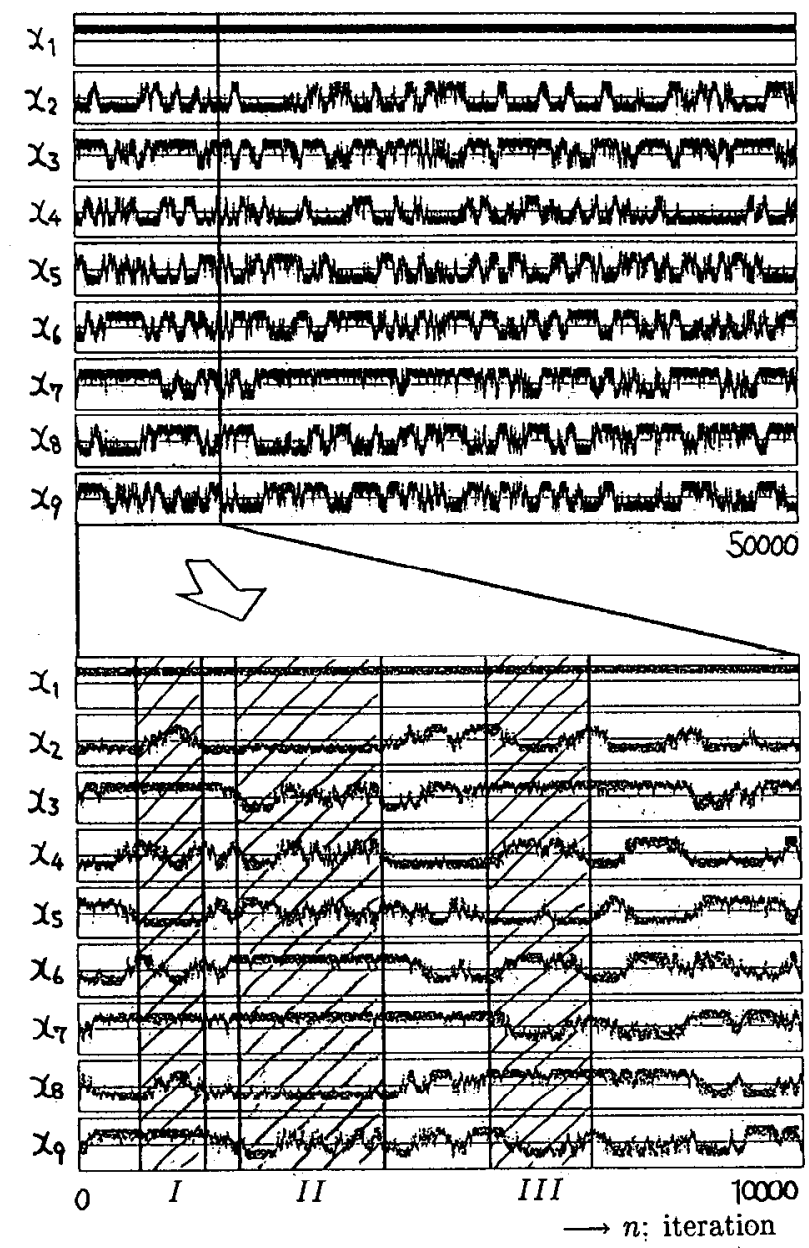

Fig. 16. Time series of Poincaré map: Self-switching of seven spatial patterns in (7) $(3 \times 3$ network). $\beta=0.298, \gamma=0.10$, and $\Delta \omega=0.0$.

chaotic network is more complicated compared with Figs. 7 or 11. This may be caused by the transmission delay. Detailed investigation on the relation between the clear switching and transmission delay is one of our important future research.
Fig. 16 shows the self-switching of the spatial patterns represented in (7) obtained from computer simulations. For example, phase states of

$$
\left(\begin{array}{ccc}
A & B & A \\
\bar{B} & \bar{A} & \bar{B} \\
A & B & A
\end{array}\right),\left(\begin{array}{ccc}
A & \bar{A} & B \\
B & \bar{B} & A \\
A & \bar{A} & B
\end{array}\right) \text { and }\left(\begin{array}{ccc}
A & B & A \\
\bar{B} & \bar{A} & \bar{B} \\
B & A & B
\end{array}\right)
$$

appear in the shaded areas I, II, and III, respectively.

This network is easily extended to large size. We can expect that various complicated spatio-temporal chaos based on the result in this section will be generated in such large scale of networks.

\section{CONCLUDING REMARKS}

In this paper, we have investigated simple autonomous chaotic circuits coupled by resistors. By carrying out computer calculations and circuit experiments, irregular self-switching phenomenon of three spatial patterns characterized by the phase states of quasi-synchronization of chaos could bc observed from only four simple chaotic circuits coupled by one resistor. We consider that this is one of the simplest spatiotemporal chaos observed from continuous dynamical systems. The six subcircuits case and the coupled chaotic circuits network were also studied, and such systems were confirmed to produce more complicated spatio-temporal phenomena.

Our future research is the theoretical approach to spatiotemporal chaos including statistical study. Extension to the large size of coupled chaotic network and the transmission delay of the change of spatial pattern in it will be also studied in the near future.

\section{ACKNOWLEDGMENT}

The authors would like to thank Professor S. Mori of Keio University and Associate Professor T. Saito of Hosei University for their valuable comments and encouragement.

\section{REFERENCES}

[1] Y. Ohmori, M. Nakagawa, and T. Saito, "Mutual coupling of oscillators with chaos and period doubling bifurcation," in Proc. ISCAS'86, 1986, pp. 61-64.

[2] L. M. Pecora and T. L. Carroll, "Synchronization in chaotic systems," Phys. Rev. Lett., vol. 64, no. 8, pp. 821-824, 1990.

[3] M. J. Ogorzalek, "Taming chaos-Part I: Synchronization," IEEE Trans. Circuits Syst. I, vol. 40, no. 10, pp. 693-699, Oct. 1993.

[4] Y. Nishio, K. Suzuki, S. Mori, and A. Ushida, "Synchronization in mutually coupled chaotic circuits," in Proc. ECCTD'93, Sept. 1993, pp. $637-642$.

[5] Y. Nishio and A. Ushida, "Multimode chaos in two coupled chaotic oscillators with hard nonlinearities," in Proc. ISCAS'94, May 1994, vol. 6, pp. 109-112.

[6] , "On a ring of chaotic circuits coupled by inductors," IEICE Trans. Fundamentals, vol. E78-A, no. 5, pp. 608-617, May 1995.

[7] , "Quasi-synchronization phenomena in chaotic circuits coupled by one resistor," to be published in IEEE Trans. Circuits Syst. I.

[8] K. Kaneko, "Period-doubling of kink-antikink patterns, quasiperiodicity in antiferro-like structures and spatial intermittency in coupled logistic lattice," Progress of Theoretical Physics, vol. 72, no. 3, pp. 480-486, Sept. 1984.

[9] _ "Spatiotemporal intermittency in coupled map lattices," Progress of Theoretical Physics, vol. 74, no. 5, pp. 1033-1044, Nov. 1985. 
[10] __ "Pattern dynamics in spatiotemporal chaos," Physica D, vol. 34, pp. $1-41,1989$.

[11] , "Clustering, coding, switching, hierarchical ordering, and control in a network of chaotic elements," Physica D, vol. 41, pp. 137-172, 1990.

[12] I. Tsuda, E. Koerner, and H. Shimizi, "Memory dynamics in asynchronous neural networks," Progress of Theoretical Physics, vol. 78, no, 1, pp. 51-71, July 1987.

[13] M. Toyoda, M. Adachi, K. Shimizu, and K. Aihara, "Pattern dynamics of chaotic ncural networks," Tech. Rep. IEICE, vol. NC89-8, pp. 43 48, 1989.

[14] K. Aihara, T. Takabe, and M. Toyoda, "Chaotic neural networks," Physics Letr. A, vol. 144, nos. 6 and 7, pp. 333-340, 1990.

[15] V. I. Nekorkin and L. O. Chua, "Spatial disorder and wave fronts in a chain of coupled chua's circuits," Int. J. Bifurc. and Chaos, vol. 3, no. 5, pp. 1281-1291, Oct. 1993.

[16] A. P.-Muñuzuri, V. P.-Muñuzuri, V. P.-Villar, and L. O. Chua, "Spiral waves on a 2-D array of nonlinear circuits," IEEE Trans. Circuits Syst. $I$, vol. 40, no. 11, pp. 872-877, Nov. 1993.

[17] A. L. Zheleznyak and L. O. Chua, "Coexistence of low- and highdimensional spatiotemporal chaos in a chain of dissipatively coupled Chua's circuits," Int. J. Bifurc. and Chaos, vol. 4, no. 3, pp. 639-674, June 1994.

[18] Y. Nishio, S. Mori, and A. Ushida, "On coupled oscillators network-For the cellular neural network," in Proc. ISCAS'93, May 1993 , vol. 4, pp. 2327-2330.

[19] N. Inaba and S. Mori, "Chaotic phenomena in circuits with a lincar negative resistance and an ideal diode," in Proc. MWSCAS'88, Aug. 1988, pp. 211-214.
[20] Y. Nishio, N. Inaba, S. Mori, and T. Saito, "Rigorous analyses of windows in a symmetric circuit," IEEE Trans. Circuits Syst., vol. 37, no. 4, pp. 473-487, Apr. 1990.

Yoshifumi Nishio (S'91-M'93) received the B.E., M.E., and $\mathrm{Ph}$ D. degrees in electrical engineering from Keio University, Yokohama Japan, in 1988, 1990, and 1993 , respectively.

In 1993, he joined the Department of Electrical and Electronic Engineering at Tokushima University, Tokushima Japan, where he is currently an Assistant Professor. His research interests are in chaos and synchronization phenomena in nonlinear circuits.

Akio Ushida (M'76) received the B.E. and M.E. degrees in electrical engineering from Tokushima University in 1961 and 1966, respectively, and the Ph.D. degree in electrical engineering from the University of Osaka Prefecture in 1974.

He was an Associate Professor from 1973 to 1980 at Tokushima University. Since 1980, he has been a Professor with the Department of Electrical Engineering at the University. From 1974 to 1975 , he spent one year as a Visiting Scholar with the Department of Electrical Engineering and Computer Sciences at the University of California at Berkeley. His current research interests include numerical methods and computer-aided analysis of nonlinear systems. 